\title{
Dermatology
}

Rigullis

\section{Case of generalized morphea complicated with systemic sclerosis}

Soichiro Sawamura, Katsunari Makino*, Hitomi Nakashima, Takamitsu Makino, Satoshi Fukushima

Department of Dermatology and Plastic Surgery, Faculty of Life Sciences, Kumamoto University, Kumamoto, Japan

Key words: localized scleroderma, antinuclear antibody, anti-centromere antibody, hyperpigmentation

\section{*Corresponding author:}

Katsunari Makino, M.D. Ph.D.

Department of Dermatology and Plastic Surgery, Faculty of Life Sciences, Kumamoto University, 11-1 Honjo, Kumamoto, Japan, 860-8556

Tel: 81-96-373-5233 Fax: 81-96-373-5235 E-mail: katsuderma@gmail.com

Authors' contributions: SS collected the data of the patient and wrote the manuscript as first author. KM was responsible for revision as corresponding author. NH collected the data of the patient. TM and SF established the diagnosis and were responsible for revision.

Conflict of interest: None declared.

Funding sources: None. 


\section{Dear Editor,}

Localized scleroderma (LSc; morphea) is a rare fibrosing disease of the skin and underlying tissues, which is different from systemic sclerosis (SSc). ${ }^{1} \mathrm{LSc}$ is classified based on clinical presentations: circumscribed, linear, generalized, pansclerotic, and mixed morphea. ${ }^{1}$ Generalized morphea (GM), which is characterized by widespread skin lesions with multiple indurated plaques or hyperpigmentation, is a relatively rare subtype occurring in 7-9\% of patients with LSc. ${ }^{1}$ Previous studies have shown that SSc can be complicated by GM, separately from GM-like SSc. Here, we present a rare case of coexistence of LSc and SSc in which LSc skin lesions appeared before SSc became apparent.

A 49-year-old woman visited our hospital for finger stiffness and Raynaud's phenomenon for a year or two, in conjunction with hyperpigmentation on the lower limbs for approximately 5 years. She had a history of chronic thyroiditis. She had been employed as an office worker for about 30 years. Physical examination revealed skin thickening on both hands (Fig. 1A), nailfold bleeding on the left forefinger (Fig. 1B), and hyperpigmentation of the limbs. The modified Rodnan total skin thickness score was 4 . Laboratory examinations revealed almost normal results, except for an elevated serum antinuclear antibody titer of $\times 1280$ (reference range: $<\times 40$ ) and positive anti-centromere antibodies. Gastroesophageal reflux disease was found on endoscopic examination. Computed tomography did not confirm interstitial lung disease. Thus, she was diagnosed with limited cutaneous SSc (lcSSc) according to the 2013 Classification Criteria for systemic sclerosis (ACR/EULAR) and the classification proposed by LeRoy et al. ${ }^{2}$ At followed-up, an oral proton pump inhibitor for gastroesophageal reflux disease, and within 1 year, peripheral erythema (called "lilac ring") with itching around the hyperpigmentation on the thighs developed (Fig. 1C and D). Additionally, the hyperpigmented lesions gradually enlarged and became indurated. Histological findings from the left thigh biopsy showed a thickened dermis with swollen collagen bundles and mild perivascular lymphocytic infiltrates. We considered that morphea lesions occurred before the onset of $1 \mathrm{cSSc}$ and recurred during follow-up. Considering all these, we diagnosed her with GM complicated with lcSSc and treated her with systemic corticosteroid (oral prednisolone, $30 \mathrm{mg} / \mathrm{day}$ ). The possibility of GMlike SSc was excluded due to the clinical course and occupational history.

The coexistence of LSc and SSc had been previously described in 4.9-6.7\% of patients with SSc. ${ }^{3}$ The presence of anti-centromere antibody in SSc patients complicated with LSc is rare compared with in SSc patients without LSc. ${ }^{4}$ To the best of our knowledge, this is the first case of GM complicated with anti-centromere antibody positive lcSSc. ${ }^{4}$ Mizutani et al. reported a case of SSc with repeated recurrent morphea as "palindromic morphea". ${ }^{5}$ Hayashi et al. also reported recurrence of morphea lesions in a patient with SSc complicated by GM when the dose of corticosteroid was tapered. ${ }^{4}$ Therefore, our patient could have a high risk of recurrence and should undergo a more careful continuous management. 


\section{References:}

1. Laxer RM, Zulian F. Localized scleroderma. Curr Opin Rheumatol 2006;18:606-13.

2. LeRoy EC, Medsger TA Jr. Criteria for the classification of early systemic sclerosis. $J$ Rheumatol 2001;28:1573-1576.

3. Toki S, Motegi S, Yamada K, et al. Clinical and laboratory features of systemic sclerosis complicated with localized scleroderma. J Dermatol 2015;42:283-7.

4. Hayashi M, Ichiki Y, Kitajima Y. Coexistence of recurrent generalized morphea and systemic sclerosis. Acta Derm Venereol 2009;89:329-30.

5. Mizutani H, Tanaka H, Okada H, et al. Palindromic morphea: multiple recurrence of morphea lesions in a case of systemic sclerosis. J Dermatol 1992;19:298-301. 


\section{Figure legend}

Figure 1. (A) Skin thickening of both hands. (B) Nailfold bleeding on the left forefinger as seen on dermoscopy. (C) Morphea lesions on both legs. (D) Irregular indurated plaques with erythematous border ("lilac ring") on the right thigh.

\section{Figure 1}

(A)

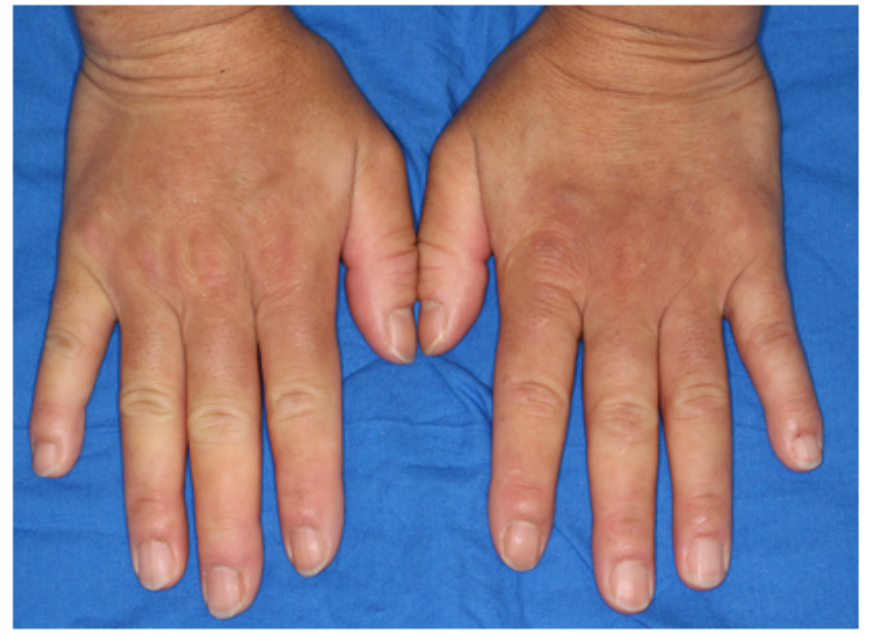

(B)

(C)

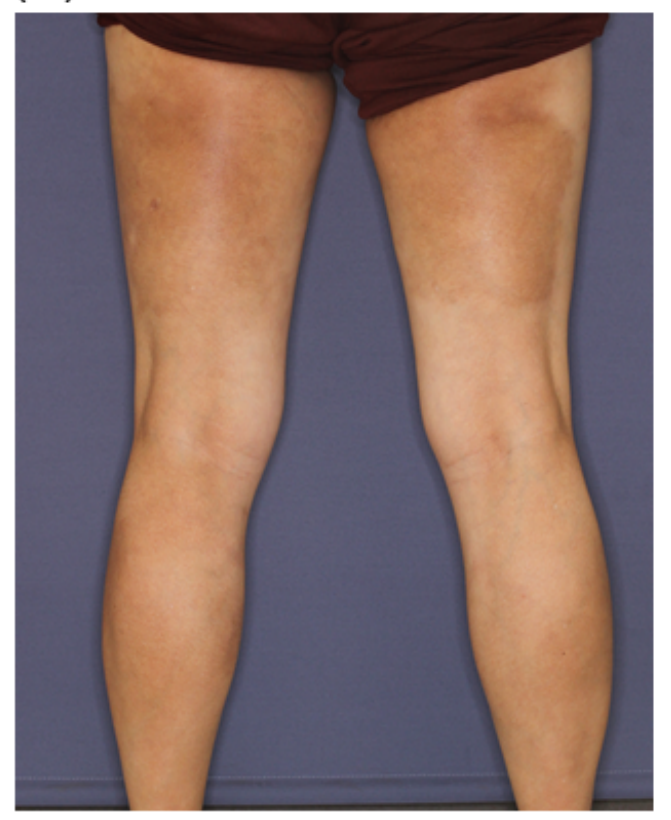

(D)

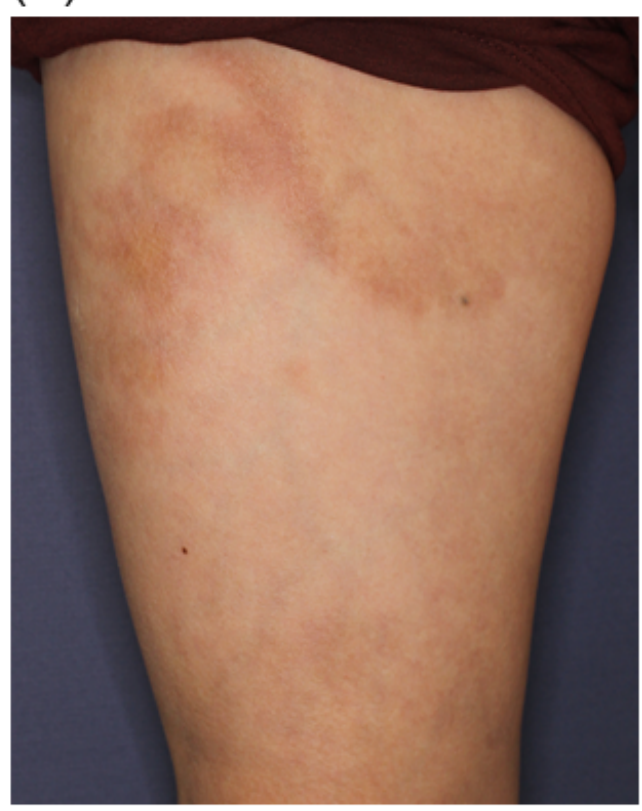

